\title{
Impact of bisphosphonate wash-out prior to teriparatide therapy in clinical practice
}

\author{
Christina Keel · Marius E. Kraenzlin • \\ Claude A. Kraenzlin · Beat Müller · \\ Christian Meier
}

Received: 22 December 2008/Accepted: 11 May 2009/Published online: 17 June 2009

(C) The Japanese Society for Bone and Mineral Research and Springer 2009

\begin{abstract}
Concurrent use of bisphosphonate therapy reduces the anabolic effect of teriparatide. Consequently, in clinical practice bisphosphonates are discontinued and teriparatide therapy held for a few months to allow bone turnover to increase. We aimed to evaluate the effect of prior bisphosphonate exposure and the effect of bisphosphonate wash-out on the treatment response to teriparatide. Thirty-nine patients with primary osteoporosis (mean age $63.6 \pm 14.0$ years), including 26 patients previously treated with oral bisphosphonates (median duration 53 months) and 13 bisphosphonate-naïve patients were started on teriparatide ( $20 \mu \mathrm{g}$ daily) and followed prospectively over 12 months. The primary study outcome was change in bone formation markers (PINP, bone ALP, osteocalcin). Secondary outcomes included changes in bone resorption $(\beta \mathrm{CTX})$ and 12-month changes in BMD. Markers of bone formation increased early during teriparatide therapy and were followed by an increase in $\beta$ CTX $(p<0.001)$. The magnitude of the increase in bone markers was comparable in both patient groups irrespective of prior bisphosphonate exposure; similarly, increases in BMD after 12 months were not significantly different
\end{abstract}

C. Keel and M. E. Kraenzlin contributed equally to this work.

C. Keel · M. E. Kraenzlin · B. Müller · C. Meier $(\bowtie)$

Division of Endocrinology, Diabetes and Clinical Nutrition,

University Hospital Basel, Missionsstrasse 24, 4055 Basel,

Switzerland

e-mail: christian.meier@unibas.ch

M. E. Kraenzlin - C. A. Kraenzlin · C. Meier

Endocrine Clinic and Laboratory, Basel, Switzerland

B. Müller

Department of Internal Medicine, Kantonsspital Aarau,

Aarau, Switzerland between bisphosphonate-pretreated and bisphosphonatenaïve patients (lumbar spine 7.1 vs. $8.9 \%, p=0.58$; total hip 4.1 vs. $1.1 \%, p=0.48$ ). The response of teriparatide was not related to the duration of bisphosphonate wash-out (median duration 4.2 months). This study confirms that beneficial effects of teriparatide on intermediate bone endpoints can be translated into clinical practice with less constringent methodological circumstances than in RCTs. Furthermore, as bisphosphonate wash-out does not appear to influence the treatment effect, teriparatide therapy can be started immediately after ceasing bisphosphonate therapy and wash-out.

Keywords Osteoporosis - Bisphosphonate $\cdot$ Teriparatide . Bone turnover marker $\cdot$ Bone mineral density

\section{Introduction}

Teriparatide (human recombinant PTH 1-34) stimulates predominantly bone-forming osteoblasts. When given intermittently, teriparatide induces bone formation by increasing the rate of bone remodeling, trabecular connectivity and cortical bone thickness, ultimately resulting in improved bone strength [1-3]. Randomized trials confirmed the beneficial effect of teriparatide with reduced incidence of osteoporotic fracture in both postmenopausal women [4] and in men [5]. The anabolic effect of teriparatide is, at least in part, mirrored by changes in biochemical markers of bone turnover. Teriparatide treatment is associated with an early increase in biochemical markers of bone formation and a delayed increase in markers of bone resorption [6-8]. This "anabolic window" apparently is thought to be responsible for the PTH-induced increase in bone mass and the preservation of bone architecture. 
In many countries in Europe, including Switzerland, teriparatide use is restricted to patients with severe osteoporosis suffering incident fractures despite antiresorptive therapy. Hence, many candidates for PTH therapy are on long-term bisphosphonate therapy. Prolonged suppression of bone remodeling due to bisphosphonates may blunt the initial anabolic effects of teriparatide. This is supported by previous studies demonstrating a diminished response to recombinant PTH with respect to BMD and bone turnover in patients with concurrent [7, 9], alternate [10] or prior [11] bisphosphonate exposure. These observations led to the clinical practice opinion that bisphosphonates should be discontinued and teriparatide treatment "held" for a 6- to 12-month period to allow turnover to increase [12, 13]. However, data investigating whether prior bisphosphonate wash-out is associated with differing treatment outcome are limited [14].

Clinical trials, i.e., randomized and placebo-controlled, are recognized as the standard means of establishing efficacy of treatment agents. Due to several circumstances, however, these data may not be valid to patients presenting in clinical practice. In order to eliminate factors that might confound treatment effects, clinical trials commonly employ inclusion and exclusion criteria. In clinical practice, however, only a minority of patients with osteoporosis (about 20\%) would be accepted for inclusion in these trials [15]. In general, teriparatide patients are older, in poorer health and are likely to have more severe osteoporosis than bisphosphonate patients [16]. In randomized trials bone markers were only assessed at the end of the study period in one run, thereby decreasing intra- and interassay variability. In order to monitor anti-osteoporotic treatment in clinical practice, however, one relies on continuous analyses of clinical endpoints, including bone turnover markers. This raises the question whether the observed changes of clinical endpoints in randomized trials, such as changes in bone turnover markers, may be extrapolated to changes observed in daily routine with less constringent real-life circumstances.

The aim of this study was first to determine the effect of teriparatide in osteoporotic patients previously treated with oral bisphosphonates as compared to treatment-naïve patients and secondly to assess whether duration of bisphosphonate wash-out prior to commencement of teriparatide therapy is related to treatment outcome as assessed by changes in bone markers and bone density.

\section{Methods}

Study design and participants

This study was a prospective, open label, nonrandomized, 12-month trial examining the effects of rhPTH (1-34) in patients with primary osteoporosis including both patients previously treated with oral bisphosphonates (alendronate $70 \mathrm{mg}$ weekly or risedronate $35 \mathrm{mg}$ weekly; $n=26$ ) and treatment-naïve patients $(n=13)$. The study was conducted in a single Endocrine and Osteoporosis Outpatient Clinic, Basel, Switzerland. Patients at our center who were considered for teriparatide therapy were consecutively enrolled into the study. In Switzerland, the Federal Office of Public Health restricts the reimbursement of teriparatide to those patients who fail to respond to prolonged treatment with bisphosphonates. Nevertheless, the decision for teriparatide treatment was based on the severity of disease after individualized clinical assessment.

Participants were women $(n=20)$ and men $(n=19)$ with a prior lumbar spine or total hip BMD T-score of $\leq-2.5 \mathrm{SD}$ and/or at least one fragility fracture. Patients were excluded if osteoporosis workup revealed a secondary cause for their osteoporosis (including glucocorticoid induced osteoporosis), or if they had a history of metabolic bone disease or vitamin $\mathrm{D}$ deficiency.

All patients received daily self-administered subcutaneous injections of teriparatide $20 \mu \mathrm{g}$ in addition to daily calcium (500 mg) and vitamin D (400 IU) for 1 year. Two postmenopausal women (both in the bisphosphonate-naïve group) remained on long-term estrogen replacement therapy. The local Ethics Committee for Human Studies approved the protocol, and informed consent was obtained from all participants.

\section{Baseline and follow-up measures}

At baseline we obtained information on demographics, health history and medication use. On the study day, morning blood samples were drawn after an overnight fast, including routine chemistry and biochemical markers of bone turnover [N-terminal propeptide of type I procollagen (PINP), bone-specific alkaline phosphatise (BAP), osteocalcin (OC) and C-terminal type I collagen telopeptide telopeptide (CTX)].

Bone mineral density at the lumbar spine and hip were evaluated by dual X-ray absorptiometry (DXA) using a Lunar Expert densitometer (Lunar, Madison, WI). A single densitometer was used throughout the study. To assess the short-term precision of the system in this population, a single repeat measurement in 20 patients was performed [17]. The coefficient of variation of individual measurements was $1.1 \%$ for the spine, $1.4 \%$ for the femoral neck, $1.9 \%$ for the trochanteric region and $1.1 \%$ for the total hip.

Follow-up visits were scheduled at 1, 4-6 and 12 months after the start of teriparatide therapy. Serum biochemical tests were obtained at all visits, whereas BMD was measured before and at the end of the study after 12 months. 
Laboratory measurements

Calcium and creatinine were analyzed by standard method on an autoanalyzer (Hitachi System 704 analyzer; Roche Diagnostics, Basel, Switzerland). Serum bone-specific alkaline phosphatase (BAP) was determined by an ELISA (ALKPHASE-B ELISA, Quidel, San Diego, CA). The intra- and interassay variations were $<5.8$ and $7.6 \%$, respectively [18]. The parameters beta-CrossLaps (CTX), N-MID-Osteocalcin (OC), N-terminal propeptide of type I collagen (PINP) and intact parathyroid hormone (iPTH) were measured in serum with electrochemiluminescence immunoassays (ECLIA) on the automated analyzer Elecsys 2010 (Roche Diagnostics, Rotkreuz, Switzerland) [19, 20]. The intra- and interassay variation was $2.4-7.2 \%$ for CTX, $1.1-5.9 \%$ for OC, $1.7-4.0 \%$ for PINP and $1.7-5.5 \%$ for intact PTH, respectively. All laboratory analyses were carried out as duplicates.

\section{Statistical analysis}

The primary study outcome was the change from baseline in bone formation markers (PINP, BAP, OC) after 1, 4-6 and 12 months. Secondary outcomes included changes in serum CTX levels (after 1, 4-6 and 12 months), 12-month changes from baseline in lumbar spine and hip BMD, and comparisons of all these outcome variables between the two study groups.

Data are shown as mean \pm SD for normally distributed variables and as median and interquartile ranges for not normally distributed variables, respectively. Unpaired $t$ test (two-sided), or Mann-Whitney $U$ test in the case of nonparametric distributions, was used to identify differences among patient groups. Changes in bone marker levels during teriparatide therapy were estimated in a repeated measures ANOVA model. Correlations between change in bone marker levels and change in BMD or duration of bisphosphonate wash-out were examined by Spearman rank correlation coefficients. $p<0.05$ was considered statistically significant. Analyses were done using SPSS software Version 15.0 (SPSS Inc. Chicago, IL).

\section{Results}

Baseline characteristics

Thirty-nine consecutive patients with primary osteoporosis (20 women, 19 men; mean age $63.6 \pm 14.0$ years) have been started on teriparatide therapy and were followed for 12 months. Thirteen patients were bisphosphonate naïve, whereas 26 subjects had prior bisphosphonate use [alendronate $n=24$; risedronate $n=2$; median duration of bisphosphonate therapy 53 months (range 3114 months)]. Bisphosphonate discontinuation was not standardized; the median bisphosphonate wash-out time was 4.2 months (range 0-10 months), which did not correlate with the duration of bisphosphonate treatment $(r=0.17, p=\mathrm{ns})$.

Patient characteristics at baseline, including serum levels of bone turnover markers and BMD at the lumbar spine and femoral neck, are presented in Table 1. Patients pretreated with bisphosphonates and bisphosphonate-naïve patients were similar for age, BMI, dietary calcium intake and BMD measures. As expected, the prior bisphosphonate group had significantly lower serum levels for biochemical markers of bone formation (PINP $p=0.01$; OC $p=0.007)$ and bone resorption (CTX $p=0.03)$. Severity of osteoporosis as assessed by number of patients with prevalent vertebral fractures (81 vs. 85\%) and mean number of vertebral fractures per patient $(2.7 \pm 1.8$ vs. $2.2 \pm 1.7)$ was comparable between pretreated and bisphosphonate-naïve patients $(p=0.95)$.

\section{Bone turnover response to teriparatide}

In response to teriparatide, overall biochemical markers of bone formation increased early in the course of therapy with peak levels after 4-6 months (PINP, BAP, OC; univariate ANOVA, $p<0.001)$ and were followed by an increase in $\beta$ CTX $(p<0.001)$ (Fig. 1). In the prior bisphosphonate group, serum levels of PINP and OC increased significantly from baseline with elevated levels at all time points during follow-up, whereas serum concentrations of CTX and BAP increased significantly only after 4-6 or 12 months, respectively. In bisphosphonatenaïve patients median changes in bone markers were numerically similar. The magnitude of the increase in all serum markers was comparable between both treatment groups irrespective of prior bisphosphonate treatment ( $p>0.05$ at all time points, Fig. 1). Specifically, absolute and relative increases of markers of bone turnover were comparable between bisphosphonate-pretreated and bisphosphonate-naïve patients, although baseline serum concentrations were significantly lower in patients with bisphosphonate pretreatment.

$\mathrm{BMD}$ response to teriparatide

Twelve months after teriparatide therapy, lumbar spine BMD increased significantly in both patients with prior bisphosphonate treatment $(p=0.001)$ and treatment-naïve patients $(p=0.015)$. Bone mineral density at the femoral neck and total hip increased in all treatment groups; however, significant changes were observed only at the femoral neck in bisphosphonate pre-treated patients $(p=0.02)$. 
Table 1 Baseline characteristics in patients starting teriparatide therapy with or without prior bisphosphonate (alendronate, risedronate) therapy

$p$ values are given for differences between patients with and without bisphosphonate treatment prior to teriparatide therapy. Values are expressed as mean \pm SD or median (IQR), as appropriate

\begin{tabular}{llll}
\hline & $\begin{array}{l}\text { Prior bisphosphonate } \\
\text { treatment }\end{array}$ & No pretreatment & $p$ value \\
\hline$N$ & 26 & 13 & \\
Age $($ years $)$ & $65.3 \pm 14.2$ & $60.5 \pm 13.6$ & 0.33 \\
BMI $\left(\mathrm{kg} / \mathrm{m}^{2}\right)$ & $23.8 \pm 6.4$ & $24.1 \pm 3.1$ & 0.86 \\
Dietary calcium $(\mathrm{mg} /$ day $)$ & $959 \pm 347$ & $977 \pm 335$ & 0.88 \\
Time since menopause (years, $n=20)$ & $18.8 \pm 13.3$ & $8.6 \pm 16.6$ & 0.19 \\
Duration bisphosphonate treatment $(\mathrm{mts})$ & $53.0(25.8,70.8)$ & - & \\
Duration bisphosphonate wash-out $(\mathrm{mts})$ & $4.2(1.3,6.8)$ & - & 0.61 \\
Calcium $(\mathrm{mmol} / \mathrm{l})$ & $2.45(2.40,2.55)$ & $2.52(2.35,2.57)$ & 0.83 \\
PTH intact $(\mathrm{pg} / \mathrm{ml})$ & $33.3(27.6,43.5)$ & $31.7(23.4,42.0)$ & 0.01 \\
S-PINP $(\mu \mathrm{g} / \mathrm{ml})$ & $24.4(18.2,35.1)$ & $42.0(23.7,67.7)$ & 0.53 \\
S-Bone ALP $(\mu \mathrm{g} / \mathrm{l})$ & $20.4(11.7,30.7)$ & $17.6(14.2,31.6)$ & 0.007 \\
S-OC $(\mathrm{ng} / \mathrm{ml})$ & $16.1(12.7,20.0)$ & $25.0(19.0,31.7)$ & 0.03 \\
S- $\beta$ CTX $(\mathrm{ng} / \mathrm{ml})$ & $0.19(0.14,0.29)$ & $0.35(0.13,0.45)$ & 0.92 \\
BMD spine $\left(\mathrm{g} / \mathrm{cm}^{2}\right)$ & $0.797 \pm 0.118$ & $0.793 \pm 0.108$ & 0.08 \\
BMD femoral neck $\left(\mathrm{g} / \mathrm{cm}^{2}\right)$ & $0.720 \pm 0.117$ & $0.789 \pm 0.106$ & 1.00 \\
Vertebral fractures $($ number of patients, \%) & $21(81 \%)$ & $11(85 \%)$ & 0.95 \\
Vertebral fractures $(\mathrm{mean}$ number of fx per & $2.7 \pm 1.8$ & $2.2 \pm 1.7$ & \\
patient) & & & \\
\hline
\end{tabular}
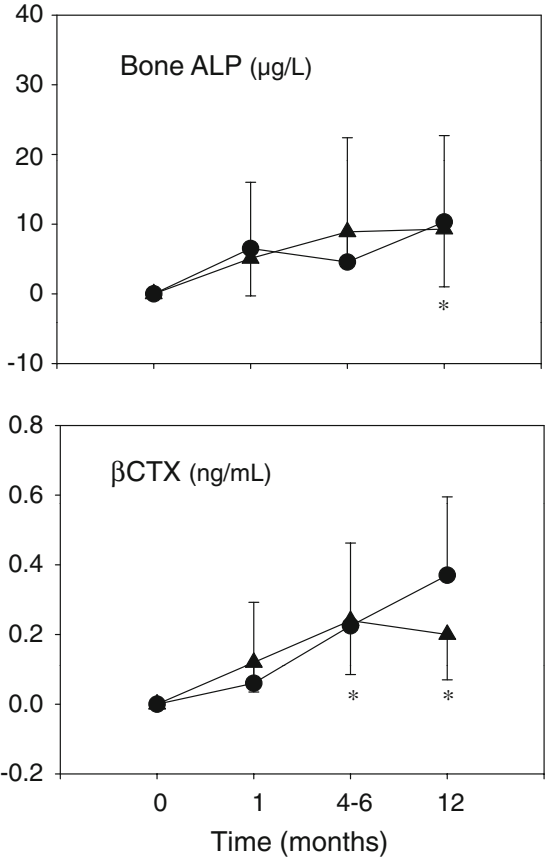

Fig. 1 Median change in serum markers of bone formation (PINP, BAP, OC) and bone resorption (CTX) during teriparatide treatment in patients with prior bisphosphonate therapy (filled triangle, $n=26$ ) and bisphosphonate-naïve patients (filled circle, $n=13$ ). Error bars indicate 25 and $75 \%$ interquartile range. $* p<0.05$ change from baseline; there were no significant differences between treatment groups at all time points

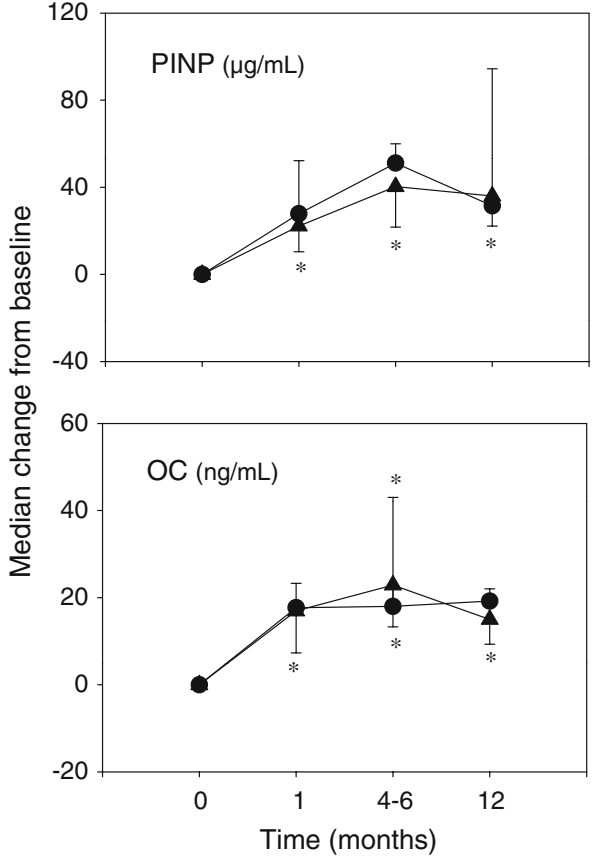

Mean percent changes in lumbar spine BMD were not significantly different between the bisphosphonate-treated patients and the bisphosphonate-naïve patients (7.1 vs. $8.9 \%, p=0.58$, Fig. 2). Similarly, increases in BMD at the total hip (4.1 vs. $1.1 \%, p=0.48$ ) and femoral neck (6.5 vs. $1.6 \%, p=0.24)$ were comparable in both treatment groups. Increases in BMD did not correlate with the observed changes in bone marker levels during teriparatide therapy in either group, nor were they associated with bone marker levels at baseline.

Correlations between duration of bisphosphonate wash-out and teriparatide response

To evaluate a potential influence of the duration of bisphosphonate wash-out prior to the initiation of teriparatide 


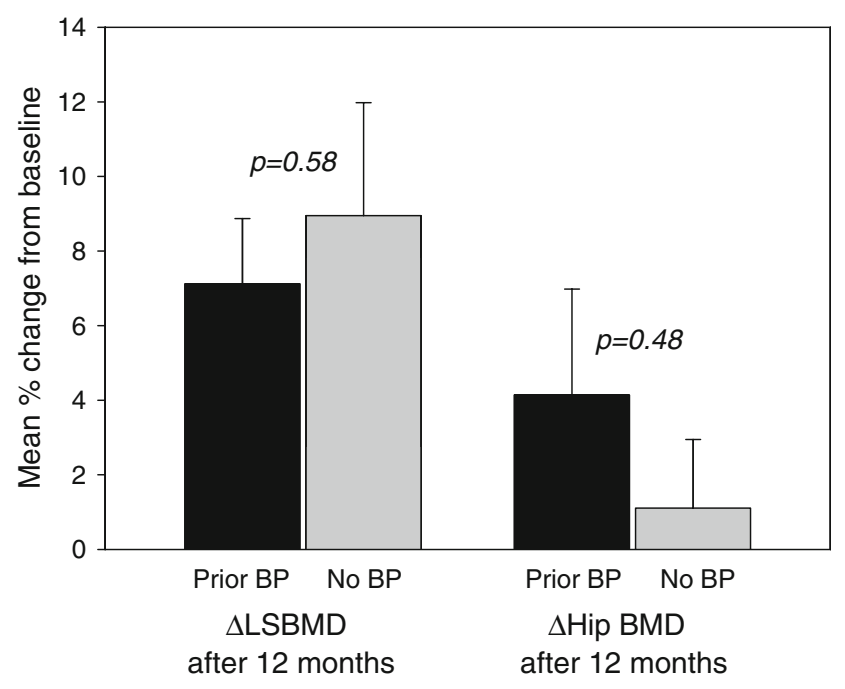

Fig. 2 Mean percent change from baseline in BMD at the lumbar spine and hip during teriparatide treatment in patients with prior bisphosphonate therapy and bisphosphonate-naïve patients. Mean \pm SEM

therapy, pretreated patients $(n=26)$ were divided in tertiles according to their duration of the bisphosphonate wash-out time (less than 2, 2-6 months and more than 6 months). Irrespective of the duration, bisphosphonate wash-out increases in serum marker of bone formation and bone resorption after 4-6 months (Fig. 3) and increases in BMD at the spine and the femoral neck after 12 months (Fig. 4) were similar in all tertile groups ( $p=\mathrm{ns}$ for trend among tertiles). Correlation data showed no significant association between the duration of bisphosphonate washout and the increase in bone turnover markers either after 1 , 4-6 or 12 months (except for an inverse correlation between bisphosphonate wash-out time and the change in CTX after 12 months; $r=-0.45, p=0.03$ ).

\section{Safety}

Three patients $(7.7 \%)$ reported side effects considered to be related to teriparatide therapy, including nausea $(n=3)$, myalgia $(n=2)$ and arthralgia $(n=1)$. Hypercalcemia during teriparatide therapy was observed in five patients $(12.8 \%)$ : one $(2.6 \%)$ treatment-naïve and four $(10.2 \%)$ with prior alendronate therapy. In all patients, hypercalcemia was considered mild and peaked off while continuing teriparatide.

\section{Discussion}

In this prospective, open-label study in postmenopausal women and in men with osteoporosis, teriparatide treatment for 12 months was associated with significant increases in bone formation markers and lumbar spine BMD irrespective of prior bisphosphonate use. Importantly, changes in bone markers and BMD were similar in patients with previous long-term bisphosphonate therapy (median duration 53 months) as compared to treatmentnaïve patients. Furthermore, in patients with prior bisphosphonates, the response to teriparatide was not related to the duration of bisphosphonate wash-out time. Our findings indicate that prior antiresorptive treatment does not substantially alter the response to teriparatide and that patients with increased fracture risk may be immediately changed to anabolic treatment after bisphosphonates have been ceased.

Teriparatide induced changes on lumbar spine BMD to a comparable extent as has been shown for treatment-naïve patients in randomized trials [4, 7]. After 12 months, areal lumbar spine BMD increased by $7.1 \%$ in patients previously treated with oral bisphosphonates and by $8.3 \%$ in treatment-naïve patients. Importantly, the increase in lumbar spine BMD was not different between the treatment groups ( $p=0.58)$, an observation that has also been made by Middleton et al. [21] in 52 postmenopausal women followed in clinical practice. Nevertheless, our results are in contrast to recent findings from another open-label clinical trial indicating that patients previously treated with bisphosphonates may have blunted BMD response to teriparatide [22]. The mean gain in spine BMD over 12 and 24 months in the EUROFORS Study was significantly greater in the treatment-naïve group (11.1 and $13.1 \%$, respectively) than in the group of patients pretreated with antiresorptives (8.6 and $10.2 \%$, respectively). Despite similar baseline characteristics differences in BMD response may be attributed to differences in study design. Obermayer-Pietsch et al. enrolled postmenopausal women only and allowed patients to be treated with various antiresorptive agents, including intravenous bisphosphonates. Furthermore, the median duration of oral bisphosphonate pretreatment was 5-26 months for alendronate and 520 months for risedronate as compared to 53 months in our study, which may account further for differences in study outcomes. Nevertheless, due to the smaller size, our study may be underpowered to detect a difference in BMD response between bisphosphonate-pretreated and treatment-naïve patients.

Previous studies have reported that teriparatide-induced changes at skeletal sites with a high proportion of cortical bone are less than at sites with predominantly trabecular bone $[9,11,23,24]$. It is assumed that at cortical sites teriparatide induces the simultaneous periostal apposition of new bone matrix and endosteal resorption of old bone matrix. Hence, patients on antiresorptives may experience more pronounced early decreases in hip BMD after starting teriparatide [14]. In part, this might explain the modest 
Fig. 3 Changes in bone marker levels after 4-6 months of teriparatide therapy according to duration of bisphosphonate wash-out in patients with previous bisphosphonate therapy. Duration of bisphosphonate wash-out is given in tertiles of the entire cohort of patients with prior bisphosphonate use $(n=26)$, i.e., less than 2, 2-6 months and more than 6 months. Mean \pm SEM

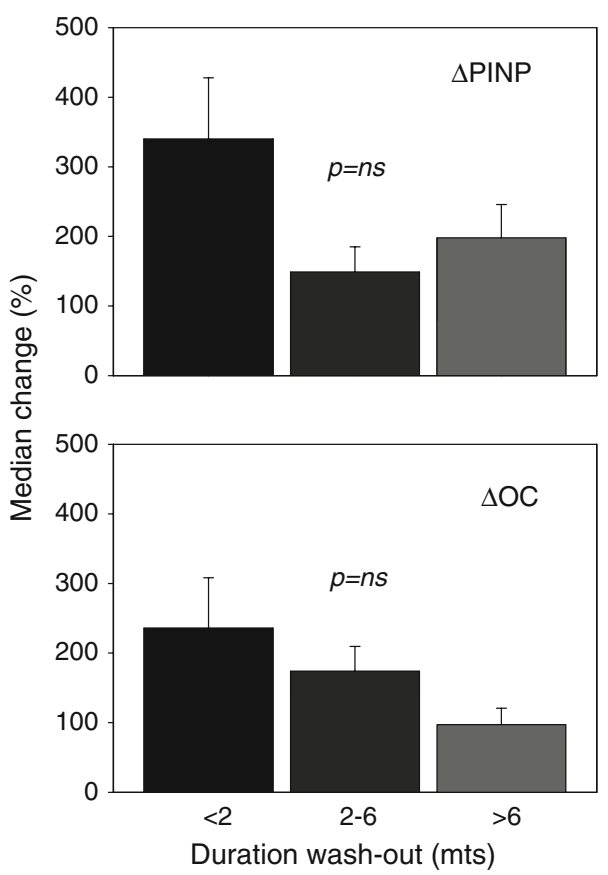

increase in femoral neck and hip BMD in our study, although we did not observe a transient decrease in hip BMD during the first 12 months of teriparatide, as has been shown by others [14, 22, 25].

Early changes in bone turnover markers with PTH therapy in treatment-naïve patients have been shown to be associated with greater increases in areal [26, 27] and volumetric [28] BMD. In this respect we were interested to know whether prior bisphosphonate therapy may influence the teriparatide response on bone markers. In fact, concurrent or prior alendronate therapy has been shown to attenuate the short-term increase in bone formation markers $[7,11]$. In our study we observed a significant anabolic effect of teriparatide on bone turnover markers regardless of previous long-term exposure to bisphosphonate treatment. Specifically, bone formation markers increased comparably during teriparatide therapy in both pretreated and treatment-naïve patients, although patients with previous bisphosphonate therapy had significantly lower bone marker levels at baseline. An early and robust increase in bone formation markers was seen already within the first 6 months of treatment. As expected, changes in formation markers were followed by an increase in serum CTX levels in accordance with findings from randomized trials [24, 29-31]. Similarly, Middleton et al. observed no difference in the teriparatide response on serum PINP levels after 3 and 6 months, although a trend towards a smaller increase in patients with prior bisphosphonate therapy as compared to treatment-naïve patients has been observed. Overall, bisphosphonate exposure did not prevent a significant increase in bone formation markers (including serum PINP) during the first months of teriparatide therapy.

Irrespective of prior antiresorptive treatment, short-term increases in bone formation markers after 1-6 months did not correlate with the observed changes in BMD after 12 months of teriparatide therapy. Whereas our findings are in contrast to previously mentioned studies in treatment-naïve patients, they are consistent with reports showing a lack in the association between increase in bone formation markers and BMD in previous alendronate or risedronate users $[11,25]$. Although we did not observe an influence of prior bisphosphonate treatment on the anabolic response to lumbar spine $\mathrm{BMD}$ we cannot exclude that the full relevance of early bone marker changes to the change in BMD may be only apparent after prolonged teriparatide treatment, i.e., 18-24 months. Importantly, no studies are available investigating the efficacy of teriparatide in reducing fracture risk in relation to early changes in surrogate markers and the potential influence of previous long-term antiresorptive therapy.

Due to the observation that teriparatide response may be blunted in previous bisphosphonate users, some clinicians advocate that teriparatide treatment should be withheld for 6 to 12 months after stopping bisphosphonates to allow bone turnover to recover $[12,13]$. Acknowledging that the suppressive effect of bisphosphonates may last for several months after treatment withdrawal [32] and the fact that the risk of incident fractures in patients with severe osteoporosis may increase questions the need for a bisphosphonate wash-out period. In fact, we found no correlation between 


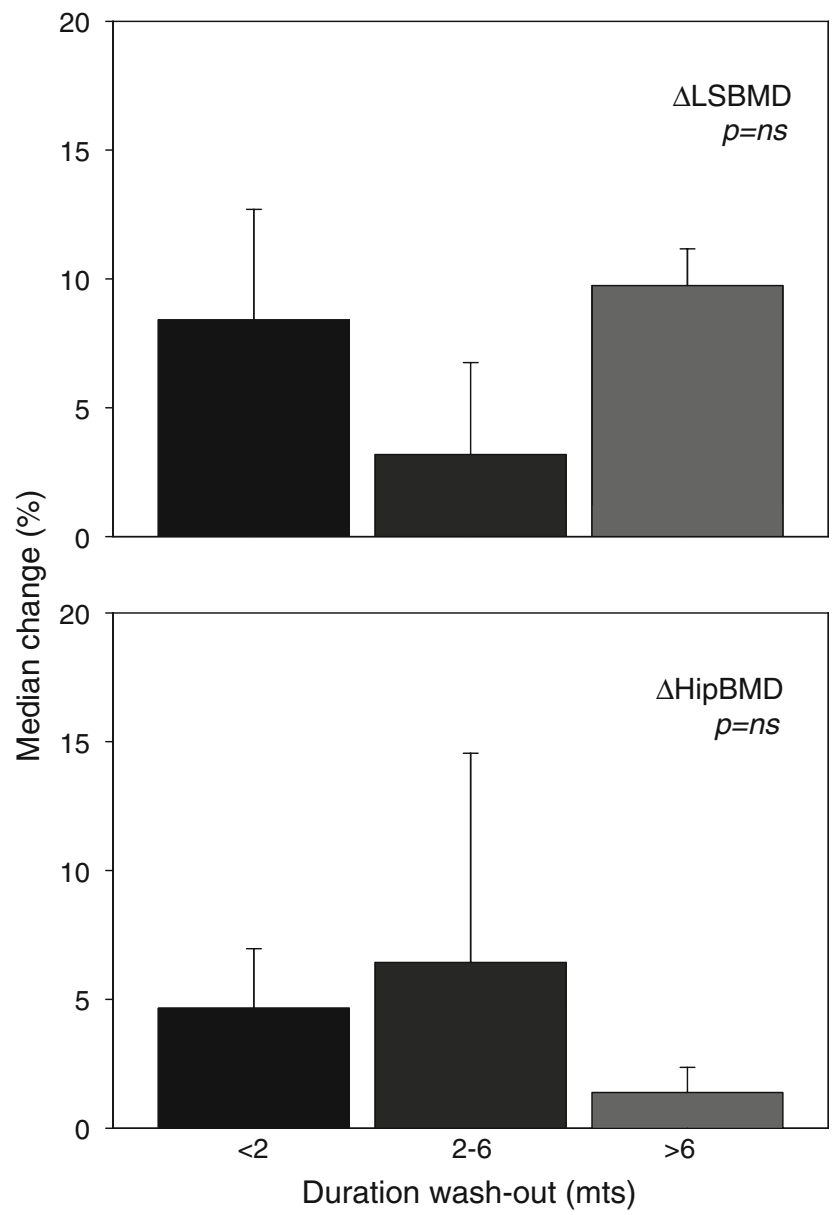

Fig. 4 Change in BMD after 12 months of teriparatide therapy according to duration of bisphosphonate wash-out in patients with previous bisphosphonate therapy. Duration of bisphosphonate washout is given in tertiles of the entire cohort of patients with prior bisphosphonate use $(n=26)$, i.e., less than 2, 2-6 months and more than 6 months. Mean \pm SEM

the duration of bisphosphonate wash-out and the early increase in bone turnover markers as well as the increase in lumbar spine and hip BMD after 12 months. The median time interval between the last bisphosphonate dose and commencement of teriparatide was 4.2 months. Nevertheless, even longer discontinuation for more than 6 months (up to 10 months) did not alter teriparatide response. As compared to previous studies, this is the first study within a clinical setting investigating longer lag times between stopping bisphosphonates and starting teriparatide. In studies by Middleton et al. [21] and Boonen et al. [14] most patients initiated teriparatide therapy within 1 month after discontinuing antiresorptive treatment. In the latter study, the duration of bisphosphonate wash-out was not associated with BMD increase [14]; however, each additional week of lag time before starting teriparatide was associated with a $0.9 \%$ increase in PINP levels and $0.4 \%$ increase in BAP levels. In view of our negative findings with longer bisphosphonate washout periods, the rather small changes in marker levels within the first weeks before starting teriparatide in the study by Boonen et al. and the lack of any association with BMD changes may question the clinical significance of this observation. With these findings we suggest that teriparatide treatment may be started immediately after bisphosphonates have been stopped without the need for prior bisphosphonate washout.

The present study's findings should be interpreted within the context of its strengths and limitations. Importantly this study assessed teriparatide response in consecutive patients who had substantial prior oral bisphosphonate treatment for a longer duration as compared to patients in the EUROFORS Study (median duration 53 vs. 20-26 months) [22]. Our study is limited by its non-randomized, open-label design; however, this resembles more accurately the reallife circumstances one would see in clinical practice, enhancing its clinical validity. Secondly, the use of bisphosphonate therapy before commencing teriparatide was not randomized, and patients with both alendronate and risedronate have been included. Due to the low number of patients overall, and specifically, the low numbers of patients on prior risedronate treatment $(n=2)$, subgroup analyses to evaluate differences in anabolic responsiveness to teriparatide in relation to the type and antiresorptive potency of prior bisphosphonate exposure are not feasible. Furthermore, due to unbalanced gender distribution within the patient groups, we were not in a position to test whether gender differences might have affected the study findings. Thirdly, we did not assess adherence of prior bisphosphonate therapy and are therefore unable to exclude irregularities in drug intake affecting subsequent teriparatide response. At baseline, however, patients with prior bisphosphonate therapy had significantly lower bone turnover marker levels as compared to treatment-naïve patients, indicating accurate compliance to prior treatment regimens. Finally, our study is not powered to assess the effect of prior bisphosphonate use or the effect of bisphosphonate washout on fracture rates or to identify subtle differences in BMD or markers of bone remodeling.

In conclusion, treatment with teriparatide for 12 months is associated with significant changes in bone formation markers and in lumbar spine BMD regardless of prior longterm bisphosphonate treatment. No blunting of teriparatide response was observed within the first year of therapy. Importantly the change in bone marker levels (within 6 months) and the change in BMD (after 12 months) was independent from the lag time between discontinuation of bisphosphonates and the initiation of teriparatide therapy. With these findings we suggest that teriparatide treatment may be started immediately after stopping bisphosphonates. 
Acknowledgments We thank Ursula Gunzenhauser, Christine Plüss and Monika Stutz for technical assistance. Preliminary data presented at the 34th European Symposium on Calcified Tissues, Copenhagen, Denmark, 5-9 May 2007. This study was supported by an unrestricted educational grant from Eli Lilly (Suisse) SA.

\section{References}

1. Seeman E, Delmas PD (2001) Reconstructing the skeleton with intermittent parathyroid hormone. Trends Endocrinol Metab 12:281-283

2. Jiang Y, Zhao JJ, Mitlak BH, Wang O, Genant HK, Eriksen EF (2003) Recombinant human parathyroid hormone (1-34) [teriparatide] improves both cortical and cancellous bone structure. J Bone Miner Res 18:1932-1941

3. Dempster DW, Cosman F, Kurland ES, Zhou H, Nieves J, Woelfert L, Shane E, Plavetic K, Muller R, Bilezikian J, Lindsay R (2001) Effects of daily treatment with parathyroid hormone on bone microarchitecture and turnover in patients with osteoporosis: a paired biopsy study. J Bone Miner Res 16:1846-1853

4. Neer RM, Arnaud CD, Zanchetta JR, Prince R, Gaich GA, Reginster JY, Hodsman AB, Eriksen EF, Ish-Shalom S, Genant HK, Wang O, Mitlak BH (2001) Effect of parathyroid hormone (1-34) on fractures and bone mineral density in postmenopausal women with osteoporosis. N Engl J Med 344:1434-1441

5. Kaufman JM, Orwoll E, Goemaere S, San Martin J, Hossain A, Dalsky GP, Lindsay R, Mitlak BH (2005) Teriparatide effects on vertebral fractures and bone mineral density in men with osteoporosis: treatment and discontinuation of therapy. Osteoporos Int 16:510-516

6. Orwoll ES, Scheele WH, Paul S, Adami S, Syversen U, DiezPerez A, Kaufman JM, Clancy AD, Gaich GA (2003) The effect of teriparatide [human parathyroid hormone (1-34)] therapy on bone density in men with osteoporosis. J Bone Miner Res 18:917

7. Finkelstein JS, Hayes A, Hunzelman JL, Wyland JJ, Lee H, Neer RM (2003) The effects of parathyroid hormone, alendronate, or both in men with osteoporosis. N Engl J Med 349:1216-1226

8. Chen P, Miller PD, Delmas PD, Misurski DA, Krege JH (2006) Change in lumbar spine BMD and vertebral fracture risk reduction in teriparatide-treated postmenopausal women with osteoporosis. J Bone Miner Res 21:1785-1790

9. Black DM, Bilezikian JP, Ensrud KE, Greenspan SL, Palermo L, Hue T, Lang TF, McGowan JA, Rosen CJ (2005) One year of alendronate after one year of parathyroid hormone (1-84) for osteoporosis. N Engl J Med 353:555-565

10. Cosman F, Nieves J, Zion M, Woelfert L, Luckey M, Lindsay R (2005) Daily and cyclic parathyroid hormone in women receiving alendronate. N Engl J Med 353:566-575

11. Ettinger B, San Martin J, Crans G, Pavo I (2004) Differential effects of teriparatide on BMD after treatment with raloxifene or alendronate. J Bone Miner Res 19:745-751

12. Miller PD, Bilezikian JP, Deal C, Harris ST, Ci RP (2004) Clinical use of teriparatide in the real world: initial insights. Endocr Pract 10:139-148

13. Canalis E, Giustina A, Bilezikian JP (2007) Mechanisms of anabolic therapies for osteoporosis. N Engl J Med 357:905-916

14. Boonen S, Marin F, Obermayer-Pietsch B, Simoes ME, Barker C, Glass EV, Hadji P, Lyritis G, Oertel H, Nickelsen T, McCloskey EV (2008) Effects of previous antiresorptive therapy on the bone mineral density response to two years of teriparatide treatment in postmenopausal women with osteoporosis. J Clin Endocrinol Metab 93:852-860
15. Dowd R, Recker RR, Heaney RP (2000) Study subjects and ordinary patients. Osteoporos Int 11:533-536

16. Foster SA, Foley KA, Meadows ES, Johnston JA, Wang S, Pohl GM, Long SR (2008) Characteristics of patients initiating teriparatide for the treatment of osteoporosis. Osteoporos Int 19:373377

17. Gluer CC, Blake G, Lu Y, Blunt BA, Jergas M, Genant HK (1995) Accurate assessment of precision errors: how to measure the reproducibility of bone densitometry techniques. Osteoporos Int 5:262-270

18. Gomez B Jr, Ardakani S, Ju J, Jenkins D, Cerelli MJ, Daniloff GY, Kung VT (1995) Monoclonal antibody assay for measuring bone-specific alkaline phosphatase activity in serum. Clin Chem 41:1560-1566

19. Garnero P, Borel O, Delmas PD (2001) Evaluation of a fully automated serum assay for C-terminal cross-linking telopeptide of type I collagen in osteoporosis. Clin Chem 47:694-702

20. Schmidt-Gayk H, Spanuth E, Kotting J, Bartl R, Felsenberg D, Pfeilschifter J, Raue F, Roth HJ (2004) Performance evaluation of automated assays for beta-CrossLaps, N-MID-Osteocalcin and intact parathyroid hormone (BIOROSE Multicenter Study). Clin Chem Lab Med 42:90-95

21. Middleton ET, Steel SA, Doherty SM (2007) The effect of prior bisphosphonate exposure on the treatment response to teriparatide in clinical practice. Calcif Tissue Int 81:335-340

22. Obermayer-Pietsch BM, Marin F, McCloskey EV, Hadji P, Farrerons $\mathrm{J}$, Boonen $\mathrm{S}$, Audran $\mathrm{M}$, Barker $\mathrm{C}$, Anastasilakis $\mathrm{AD}$, Fraser WD, Nickelsen T (2008) Effects of two years of daily teriparatide treatment on BMD in postmenopausal women with severe osteoporosis with and without prior antiresorptive treatment. J Bone Miner Res 23:1591-1600

23. Keaveny TM, Donley DW, Hoffmann PF, Mitlak BH, Glass EV, San Martin JA (2007) Effects of teriparatide and alendronate on vertebral strength as assessed by finite element modeling of QCT scans in women with osteoporosis. J Bone Miner Res 22:149-157

24. Black DM, Greenspan SL, Ensrud KE, Palermo L, McGowan JA, Lang TF, Garnero P, Bouxsein ML, Bilezikian JP, Rosen CJ (2003) The effects of parathyroid hormone and alendronate alone or in combination in postmenopausal osteoporosis. N Engl J Med 349:1207-1215

25. Miller PD, Delmas PD, Lindsay R, Watts NB, Luckey M, Adachi J, Saag K, Greenspan SL, Seeman E, Boonen S, Meeves S, Lang TF, Bilezikian JP (2008) Early responsiveness of women with osteoporosis to teriparatide following therapy with alendronate or risedronate. J Clin Endocrinol Metab 93:3785-3793

26. Chen P, Satterwhite JH, Licata AA, Lewiecki EM, Sipos AA, Misurski DM, Wagman RB (2005) Early changes in biochemical markers of bone formation predict BMD response to teriparatide in postmenopausal women with osteoporosis. J Bone Miner Res 20:962-970

27. Bauer DC, Garnero P, Bilezikian JP, Greenspan SL, Ensrud KE, Rosen CJ, Palermo L, Black DM (2006) Short-term changes in bone turnover markers and bone mineral density response to parathyroid hormone in postmenopausal women with osteoporosis. J Clin Endocrinol Metab 91:1370-1375

28. Dobnig H, Sipos A, Jiang Y, Fahrleitner-Pammer A, Ste-Marie LG, Gallagher JC, Pavo I, Wang J, Eriksen EF (2005) Early changes in biochemical markers of bone formation correlate with improvements in bone structure during teriparatide therapy. J Clin Endocrinol Metab 90:3970-3977

29. Lindsay R, Nieves J, Formica C, Henneman E, Woelfert L, Shen V, Dempster D, Cosman F (1997) Randomised controlled study of effect of parathyroid hormone on vertebral-bone mass and fracture incidence among postmenopausal women on oestrogen with osteoporosis. Lancet 350:550-555 
30. Kurland ES, Cosman F, McMahon DJ, Rosen CJ, Lindsay R, Bilezikian JP (2000) Parathyroid hormone as a therapy for idiopathic osteoporosis in men: effects on bone mineral density and bone markers. J Clin Endocrinol Metab 85:3069-3076

31. Hodsman AB, Hanley DA, Ettinger MP, Bolognese MA, Fox J, Metcalfe AJ, Lindsay R (2003) Efficacy and safety of human parathyroid hormone-(1-84) in increasing bone mineral density in postmenopausal osteoporosis. J Clin Endocrinol Metab 88:5212-5220
32. Black DM, Schwartz AV, Ensrud KE, Cauley JA, Levis S, Quandt SA, Satterfield S, Wallace RB, Bauer DC, Palermo L, Wehren LE, Lombardi A, Santora AC, Cummings SR (2006) Effects of continuing or stopping alendronate after 5 years of treatment: the fracture intervention trial long-term extension (FLEX): a randomized trial. JAMA 296:2927-2938 